\title{
The Effect of Shock Wave Strength on Shock Bubble Interaction
}

\author{
Jie YANG ${ }^{\mathrm{a}}$, Zhen-Hua WAN ${ }^{\mathrm{b}}$, De-Jun SUN ${ }^{\mathrm{c}, *}$
}

Department of Modern Mechanics, University of Science and Technology of China, Hefei 230027, China

\author{
ayjyang@mail.ustc.edu.cn, bwanzh@ustc.edu.cn, cdsun@ustc.edu.cn \\ ${ }^{*}$ Corresponding author
}

Keywords: Piecewise Parabolic Method, Shock-bubble Interaction, Mixing.

\begin{abstract}
The three-dimensional shock-bubble interaction is investigated numerically using high-resolution piecewise parabolic method, in which both the viscous and turbulence effects are considered. The bubble is fixed at the same size and is accelerated by a planar shock wave of different strengths, in which the Mach number (Ma) ranges from 1.2 to 6.0. The bubbles are filled with four different gases (Helium, Nitrogen, Krypton and R12) surrounded by air in order to investigate different density jumps across the interface (Atwood number $-0.8<\mathrm{A}<0.7$ ). The computational results of low Ma cases agree well with that of experiments quantitatively. The deformation process of bubble geometry is quite different as the change of A and Ma, particularly for $\mathrm{A}>0$ and $\mathrm{A}<0$. In all cases, statistical quantities like the compression ratio, mean bubble fluid velocity and mixing ratio are studied in detail. As the increase of Ma for fixed $\mathrm{A}$, the compression ratio is highly raised, and the time-dependent mean bubble velocity is influenced as well. In addition, the 'mixedness' between two fluids is enhanced greatly as Ma increases. More importantly, it is found that some existed scaling laws of these quantities for the shock wave strength can't be directly applied to high Ma cases.
\end{abstract}

\section{Introduction}

The problem of a shock interact with a bubble has received wide concerns due to its interesting features involved, since the pioneer work of Rudinger \& Somers (1960)[1]. The fundamental features like the distribution of inhomogeneities of density, pressure, temperature or other state variables can serve as strong perturbations which will alter the shock fronts, bubble geometry and many other properties when driven by a planar shock wave impulsively[2, 3]. For instance, the gradients of density and pressure are not colinear after shock passage and act as an important source in generating characteristic vortices, and hence result in further distortion of the interface and mixing between two fluids [5]. It is well-known that the interaction of a shock with a density inhomogeneity will lead to Richtmyer-Meshkov instabilities (RMI) [6], which occur in broad area of applications such as inertial confinement fusion (ICF) implosions, combustion of scramjet or supernova explosion. There are some similarities in basic features between shock-bubble interaction (SBI) and RMI, and therefore the better understanding of the former can help us dealing with RMI. Due to the nature of multifluid mixture or even multiphase, in combination of the complicated underlying mechanisms of RMI, a lot of efforts have been made to study SBI theoretically [7], experimentally [5] and numerically [8,9].

The SBI system has many configurations, wherein the simplest one is corresponding to a single spherical bubble accelerated by a planar incident shock. The ambient unshocked gas medium has the density $\rho_{1}$, and the unshocked bubble has density $\rho_{2}$. A dimensionless parameter is defined referred to as Atwood number (A) to scale the density jump $A=\left(\rho_{2}-\rho_{1}\right) /\left(\rho_{2}+\rho_{1}\right)$. The incident shock of different intensity (usually generated in a shock tube experimentally) is scaled by Mach number Ma. A>0 means that the bubble is heavier than the ambient gas ('heave-bubble' case), which also implies that the sound speed in the bubble $c_{2}$ is faster than that in ambient gas $c_{1}$. On the contrary, $\mathrm{A}<0$ represents a 'light-bubble' case $\left(c_{1}>c_{2}\right)$. The difference of sound speed has great 
influence on the shock refraction pattern, which is a determinant associated with bubble deformation [8]. Therefore, the A and Ma are two of the most important control parameters for SBI.

\section{Numerical Method}

So far, many numerical studies of SBI have been carried out, however, relatively little work has been made to study the SBI with strong shocks in three dimensions (3D), particularly considering the viscous and turbulence effects. In this work, the gas bubble $(-0.8<\mathrm{A}<0.7)$ which is accelerated by both weak and strong shock will be simulated using high-resolution piecewise parabolic method (PPM) in 3D with viscous and turbulence effects considered. Four cases of different shock strength are considered: two 'weak-shock' cases $(\mathrm{Ma}=1.2,1.5)$ and two 'strong-shock' cases $(\mathrm{Ma}=3.0,6.0)$. We have focused on investigating the morphology and time-dependent bubble dynamics, and some integral properties have be presented quantitatively. The results of 'weak-shock' cases are compared to that of the existed experiments [5]. In addition, the integral properties of all cases are analyzed and compared so as to clarify the partial influence of Mach number.

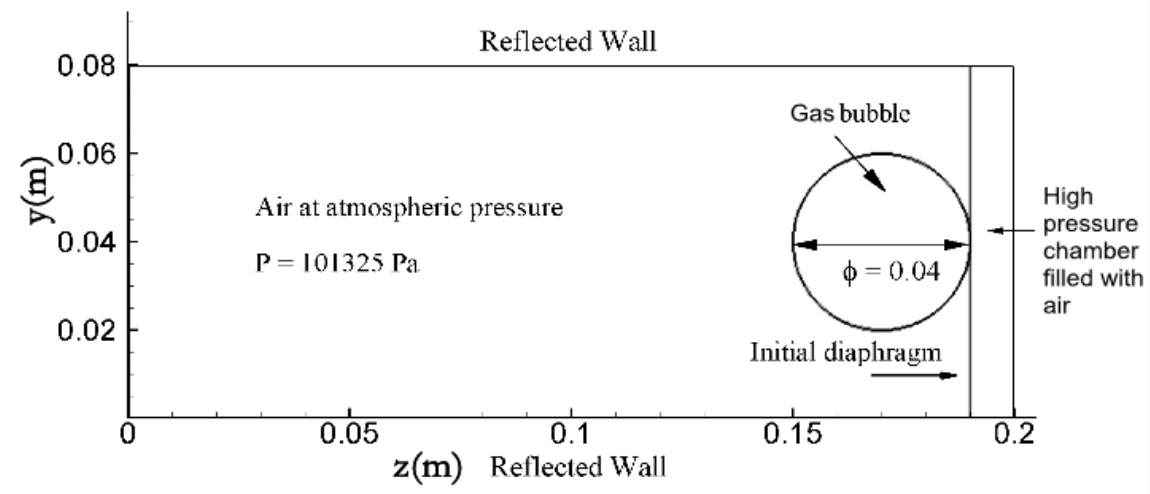

Fig. 1 the Configurations of the Computational Domain

The configuration of the computational domain is shown in Fig.1. The domain has the size of $(0.08 \times 0.08 \times 0.2) \mathrm{m}$ in $x, y, z$ direction respectively. The bubble has a diameter $\phi=0.04 \mathrm{~m}$ and its center is located at $(0.04,0.04,0.17) \mathrm{m}$. The initial diaphragm is placed at $\mathrm{z}=0.19 \mathrm{~m}$, the left is unshocked gas with atmospheric pressure $\mathrm{p}=101325 \mathrm{~Pa}$. The bubble is filled with four different gases (Helium, Nitrogen, Krypton and R12), and the ambient gas is air. The initial properties and sound speeds for the gases are given in table 1(The initial temperature is $293 \mathrm{~K}$ ).

Tab. 1 the Initial Properties of the Gases

\begin{tabular}{llccc}
\hline Gas & \multicolumn{1}{c}{$M$} & $\gamma$ & $\rho$ & $c$ \\
$\left(\mathrm{~g} \cdot \mathrm{mol}^{-1}\right)$ & & $\left(\mathrm{kg} \cdot \mathrm{m}^{3}\right)$ & $\left(\mathrm{m} \cdot \mathrm{s}^{-1}\right)$ \\
$\mathrm{Air}$ & 29.0 & 1.402 & 1.29 & 330.0 \\
$\mathrm{He}$ & 4.0 & 1.667 & 0.167 & 1005.6 \\
$\mathrm{~N}_{2}$ & 28.0 & 1.401 & 1.25 & 336.9 \\
$\mathrm{Kr}$ & 83.8 & 1.672 & 3.49 & 220.5 \\
$\mathrm{R} 12$ & 120.9 & 1.141 & 5.13 & 150.1 \\
\hline
\end{tabular}

The filtered three-dimensional Navier-Stokes equations in Cartesian coordinates are employed to resolve the this problem, which are expressed as

$$
\frac{\partial \bar{\rho}}{\partial t}+\frac{\partial \bar{\rho} \tilde{u}_{j}}{\partial x_{j}}=0
$$




$$
\begin{aligned}
& \frac{\partial \bar{\rho} \tilde{u}_{i}}{\partial t}+\frac{\partial \bar{\rho} \tilde{u}_{j} \tilde{u}_{i}}{\partial x_{j}}+\frac{\partial \bar{p}}{\partial x_{i}}=\frac{\partial\left(\bar{\sigma}_{i j}+\tau_{i j}\right)}{\partial x_{j}} \\
& \frac{\partial \bar{\rho} \bar{E}}{\partial t}+\frac{\partial\left(\bar{\rho} \tilde{u}_{j} \bar{E}+\bar{p} \tilde{u}_{j}\right)}{\partial x_{j}}=-\frac{\partial\left(\bar{q}_{j}+Q_{j}\right)}{\partial x_{j}}+\frac{\partial\left(\tilde{u}_{i}\left(\bar{\sigma}_{i j}+\tau_{i j}\right)\right)}{\partial x_{j}} \\
& \frac{\partial \bar{Y}^{(s)}}{\partial t}+\tilde{u}_{j} \frac{\partial \bar{Y}^{(s)}}{\partial x_{j}}=\frac{\partial}{\partial x_{j}}\left(\tilde{D} \frac{\partial \bar{Y}^{(s)}}{\partial x_{j}}\right), \quad s=1,2, \ldots, N-1
\end{aligned}
$$

$\bar{\sigma}_{i j}=\mu_{l}\left[\partial \tilde{u}_{i} / \partial x_{j}+\partial \tilde{u}_{j} / \partial x_{i}-2 / 3 \delta_{i j} \partial \tilde{u}_{k} / \partial x_{k}\right]$ is the viscous stress tensor, $\tau_{i j}=\rho\left(\bar{u}_{i} u_{j}-\bar{u}_{i} \bar{u}_{j}\right)$ is the subgrid scale(SGS)stress tensor which is explicitly modelled by the same techniques adopted in[10]. $\mathrm{N}$ is the number of components.

To solve the Equations (1)-(4), the inviscid fluxes are discretized by PPM method, while the viscous and heat fluxes are discretized by second-order central difference scheme. Using the dimension-splitting technique, the 3D problem can be simplified to 1D problem, and then two-step Lagrange-Remap algorithm is applied for time advancing [11]. About 37 million cells are adopted in the computation, and at least 120 cells are distributed in the bubble in each direction.

In this work, the conditions of 'weak-shock' cases are set the same to Layes \& Métayer's experiments [5] for the sake of comparison. Layes \& Métayer measured the trajectory of incident and transmitted shock, and two points on the interface indicated by point 1 and 4 , which located at the upstream structure and downstream one, respectively. The comparisons between the present results and experimental trajectories are made, and good agreement is achieved, as illustrated in Fig.2. At late times, the velocity of point 1 and 4 are almost constant, and the latter is faster.

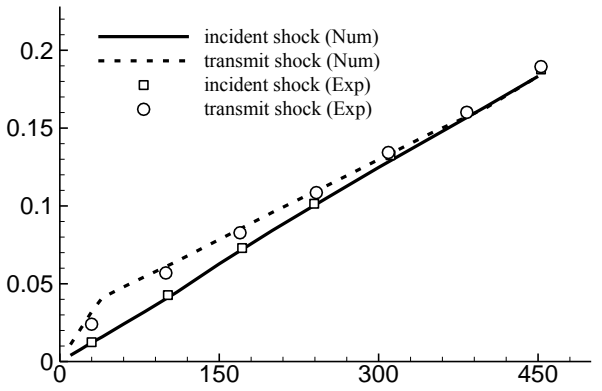

(a)

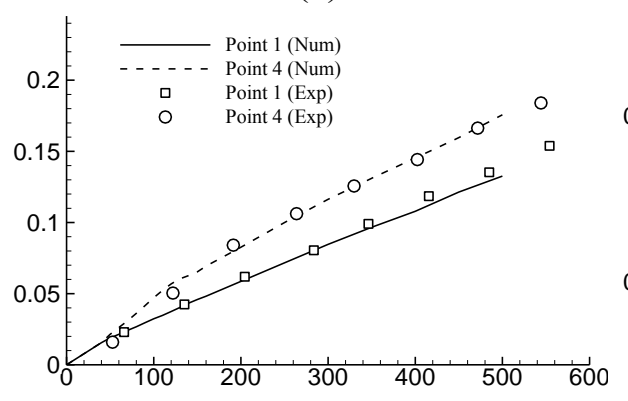

(c)

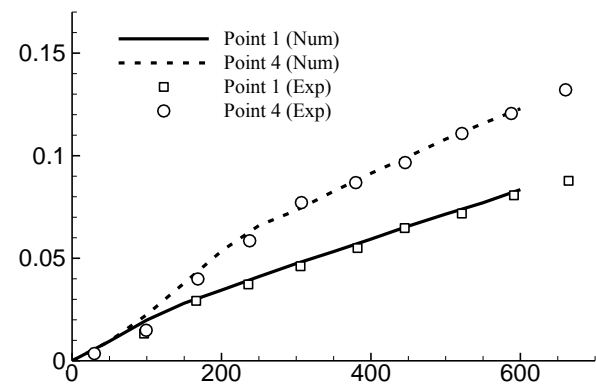

(b)

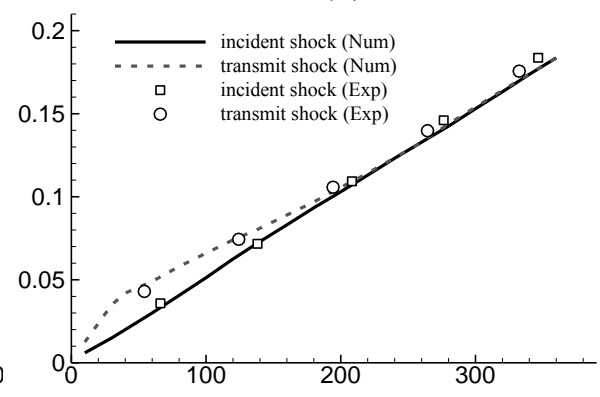

(d)

Fig. 2 the Trajectories of Numerical Simulations and Experiments (a, b) $\mathrm{Ma}=1.2 ;(\mathrm{c}, \mathrm{d}) \mathrm{Ma}=1.5$

\section{Results and Discussions}

The time-dependent morphology of the Helium bubble is shown in Fig.3. Because of $A<0$, the incident shock wave is slower than the transmitted shock wave, and the latter has convex curvature. Influenced by baroclinic mechanism, vorticity is generated and deposited on the interface due to strong gradients of density and pressure [12]. The upstream portion of the interface is inversed, and a vortex ring is formed in all cases initially. With increased Mach number, the bubble deformation 
becomes larger, and the size of the bubble is diminished greatly. A significant difference between strong and weak shock cases is found: there are two vortex rings formed in cases of $\mathrm{Ma}=1.2,1.5$ and the forward one contains more helium gas as increase of Ma while only a single vortex ring is found in 'strong-shock' cases, as shown in Fig.3.

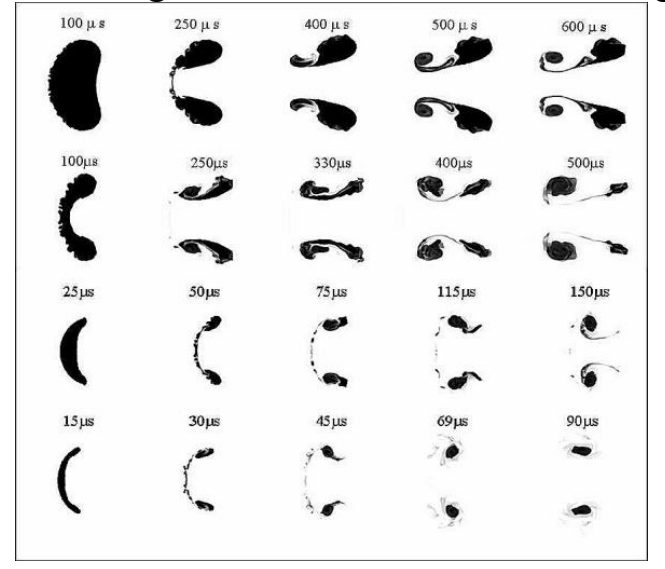

(a) The volume fraction in $y-z$ plane at $x=0.04 m$ (from top to bottom $\mathrm{Ma}=1.2,1.5,3.0$ and 6.0)
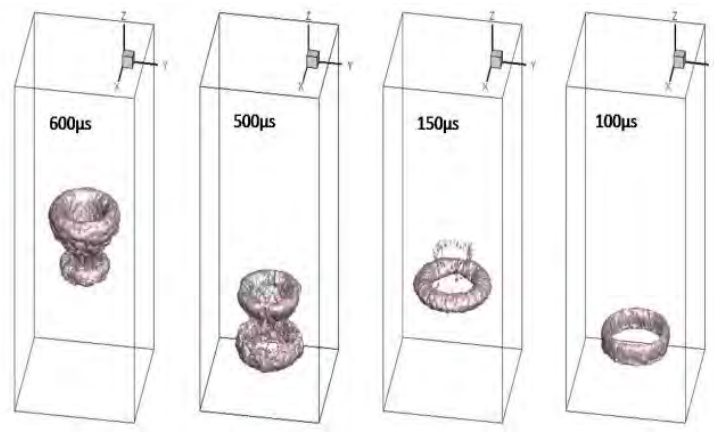

(b) The iso-surfuce of the volume fraction $\left(Y_{\text {helium }}=0.75\right)($ from left to right $\mathrm{Ma}=1.2,1.5,3.0$ and 6.0)

Fig. 3 the Time-dependent Morphology of the Helium Bubble

As aforementioned, the size of bubble decreases with increase in Ma, this is response of the bubble to the compressive effects. Such effects can be quantitatively measured through integral flow quantities, for instance, to track the mean density of the bubble fluid. Niederhaus et al.(2008)[8] measured the 'compression ratio' for different scenarios using simulation data, and then normalized them to one-dimensional model of Giodnao \& Burtschell(2006)[13]. The compression ratio is defined as $C(t)=V_{0} / V^{*}(t)$ and $\tilde{C}(t)$ is the normalized one, where $V^{*}(t)$ is the total weighted volume of the helium gas written as $V^{*}(t)=\int_{D} Y(x, y, x, t) d V . Y$ is the local volume fraction of bubble gas and $D$ is the entire computational domain. $V_{0}$ is the initial volume of the bubble.

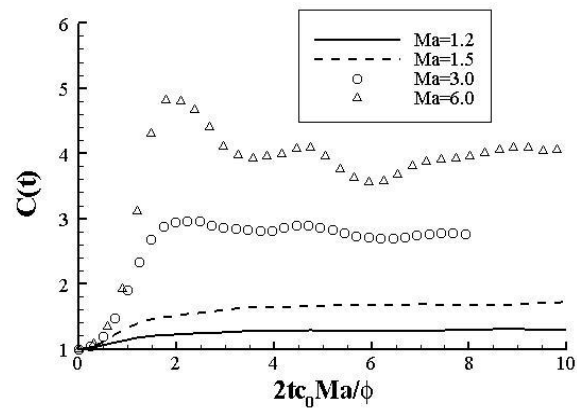

(a)

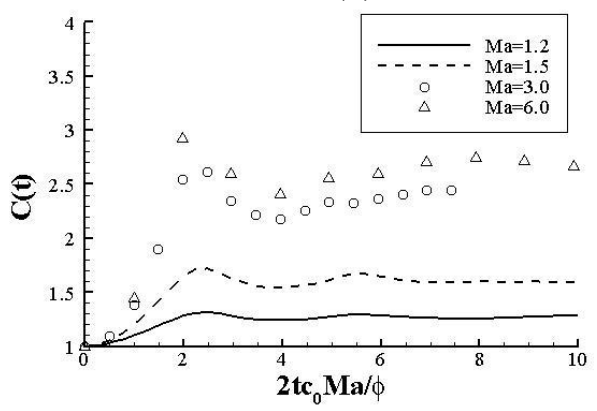

(c)

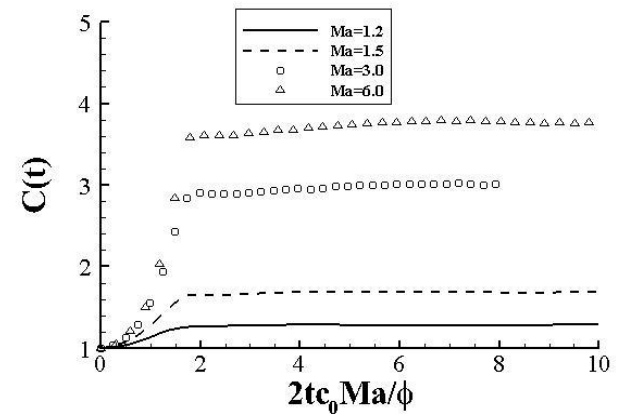

(b)

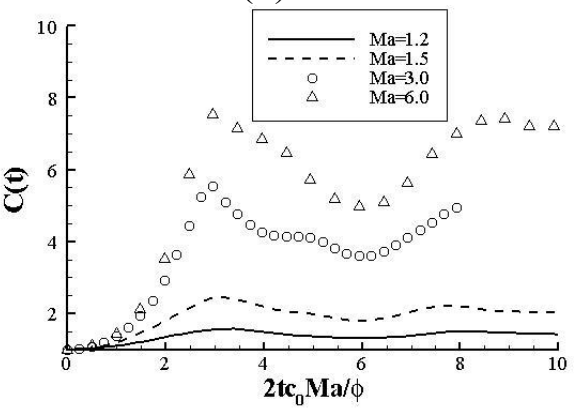

(d)

Fig. 4 Compression Ratio $C(t)$-Dimensionless Time(a)air-He; (b)air- $\mathrm{N}_{2}$; (c)air-Kr; (d)air-R12 
Fig.4 shows the history of $C(t)$ in present simulations, and the time is scaled based on $\mathrm{Ma}$ and sound speed $c_{0}$ in unshocked air. It is interesting that $C(t)$ approaches a value close to Ma for $\mathrm{Ma} \leq 3.0$. For $\mathrm{Ma}=6.0$, drastic oscillations are observed for $C(t)$, which should be related with stronger intensity of scattered shocks and rarefaction waves. In 'weak-shock' cases, the amplitudes of oscillations are much smaller. Here, in the case of Helium bubble, $\tilde{C}(t)$ is computed using densities according to [8] in Fig.5, with the final volume $V_{f}$ estimated directly in simulation at late times. It is easy to find that $\tilde{C}(t)$ yields quite well collapse for $\mathrm{Ma}=1.2$ and 1.5.For $\mathrm{Ma} \geq 3.0, \tilde{C}(t)$ grows quicker and reaches a peak value larger than 1 around dimensionless time 2 . It seems that the unified normalization can describe the low Ma cases well, but high Ma cases violate such normalization.

The mean Helium bubble fluid velocities are presented in Fig. 5(b). In the simulation, the bubble moves along z-direction, hence, the $w$ is used to compute the integral velocity: $\bar{v}(t)=\frac{1}{V_{0}} \cdot \int_{D} \rho_{b} Y w d V$, where $\rho_{b} \quad$ is the local partial density of the bubble fluid. $\bar{v}$ increases fast when accelerated by the incident shock wave at the initial stage, then oscillate and settle down to a nearly constant value gradually. Here, the inflow velocity $U_{\text {flow }}$ is utilized to scale $\bar{v}$. For $\mathrm{Ma} \leq 3.0$ the final $\bar{v}$ around $1.5 U_{\text {flow }}$, while this value is near $U_{\text {flow }}$ as $\mathrm{Ma}=6.0$. In the shadow area, $\bar{v}$ decrease abruptly in cases of $\mathrm{Ma}=3$ and 6 . The decline occurs at the moment that the transmitted shock wave leaves the bubble and the bubble is largely deformed.

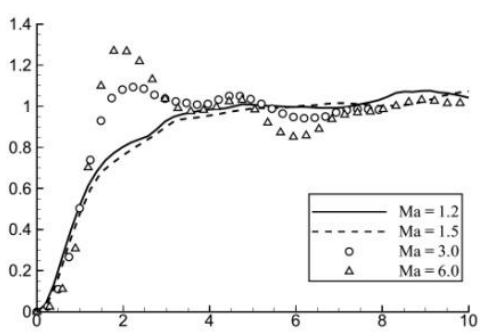

(a)

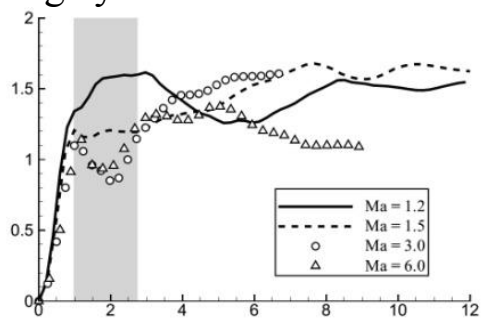

(b)

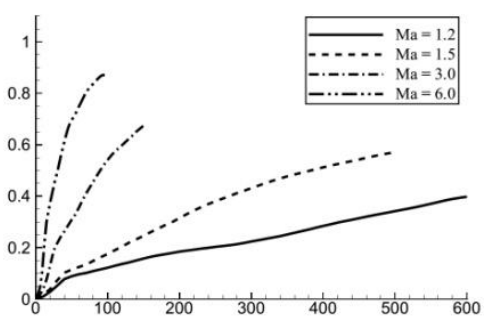

(c)

Fig. 5 Air-He: (a) .Normalized Compression Ratio $\tilde{C}(t)$ - dimensionless time (b) The mean velocities of bubble. (c)The time-dependent mixing ratio $\varsigma(t)$

An important aspect of SBI is mixing between the helium gas and air. A dimensionless quantity

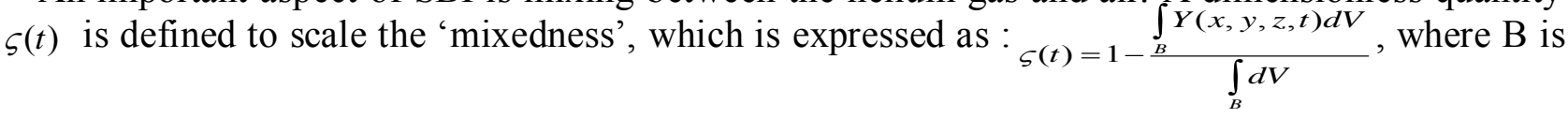
the region containing the bubble fluid. The Helium bubble time-dependent 'mixedness' is plotted in Fig.5(c). It is obvious that the 'mixedness' is highly raised with the increased Ma. The growth rate of $\varsigma(t)$ varies before and after the shock passage, the former is larger, which can easily be found in cases of $\mathrm{Ma}=1.2$ and 1.5. However, such a trend is less obvious in 'strong-shock' cases.

\section{Summary}

The interactions of a planar shock and a gas bubble in 3D with different Ma have been investigated. The high-order PPM method is utilized to capture the interface of the two fluids in consideration of viscous and turbulence effects. It is found that the bubble deforms quite different as the increase of Ma, two vortex rings are identified in low Ma cases, while only a single vortex ring is found in high Ma cases. The quantitative results measured in present simulations agree well with that of experiments. The compression ratio $C(t)$ increases as $\mathrm{Ma}$ increases, and unified normalization $\tilde{C}(t)$ can only applied to low Ma cases. In addition, Ma has a large impact on the 
mean bubble fluid velocity, as well as the 'mixedness'. The 'mixedness is highly enhanced as Ma is increased.

\section{References}

[1]Rudinger G, Somers L M , Behaviour of small regions of different gases carried in accelerated gas flows, J. Fluid Mech.7(1960)161-176.

[2]D Ranjan, J Oakley, R Bonazza, Shock-bubble interactions, Annu. Rev. Fluid Mech. 43(2011) 117-140.

[3]J Haas, B Sturtevant, Interaction of weak shock waves with cylindrical and spherical inhomogeneities, J. Fluid Mech. 181 (1987)41-76.

[4]J Quirk, S Karni, On the dynamics of a shock-bubble interaction .Fluid Mech. 318 (1996) 129-163.

[5]G Layes, O Le Métayer, Quantitative numerical and experimental studies of the shock accelerated heterogeneous bubbles motion, Phys.Fluids.19(2007)042105.

[6]M Brouillette, The Richtmyer-Meshkov instability, Annu. Rev. Fluid Mech. 34(2002) 445-468.

[7]R Saurel, S Gavrilyuk, F Renaud, A multiphase model with internal degrees of freedom: application to shock-bubble interaction, J. Fluid Mech.495 (2003)283-321.

[8]J Niederhaus, J Greenaugh, J Oakley, D Ranjan, M Anderson, R. Bonazza, A computational parameter study for the three-dimensional shock-bubble interaction, J.Fluid Mech.594 (2008)85-124.

[9]A Bagabir, D Drikakis, Mach number effects on shock-bubble interaction, Shock Waves.11 (2001)209-218.

[10]J Bai, J Liu, T Wang, L Zou, P Li, D Tan, Experimental and numerical study of shock-accelerated elliptic heavy gas cylinders, Phys. Review E.81(2010)056318.

[11]J Zheng, T Lee, A high-resolution method for compressible two-fluid flows and simulation of three-dimensional shock-bubble interactions, J. Numer. Meth. Fluids. 72 (2) (2012)206-230.

[12]J Picone, J Boris, Vorticity generation by shock propagation through bubbles in a gas, J. Fluid Mech.189 (1988)23-51.

[13]J Giordano, Y Burtschell, Richtmyer-Meshkov instability induced by shock-bubble interaction: numerical and analytical studies with experimental validation, Phys.Fluids 18(2006)036102. 